\title{
Effects of Continuous Plasma-Derived Subcutaneous C1-Esterase Inhibitor on Coagulation and Fibrinolytic Parameters
}

\author{
${ }^{1}$ Division of Allergy, Immunology, and Angioedema, Barzilai Medical \\ Center, Ashkelon, Israel \\ 2 Division of Basic and Clinical Immunology, Department of Medicine, \\ University of California at Irvine, Orange, California, United States \\ ${ }^{3}$ Department of Immunology, Addenbrooke's Hospital, Cambridge \\ and UCLH, London, United Kingdom \\ ${ }^{4}$ Ospedale Luigi Sacco/U.O. Medicina Generale, Milano, Italy \\ ${ }^{5}$ Allergy, Immunology and Respiratory Research, Department of \\ Medicine and Pediatrics, Penn State University, Hershey, \\ Pennsylvania, United States \\ ${ }^{6}$ Department of Medicine, McMaster University, Hamilton, Ontario, \\ Canada \\ ${ }^{7}$ CSL Behring GmbH, Marburg, Germany \\ ${ }^{8} \mathrm{CSL}$ Behring GmbH, Marburg, Germany (former employee)
}

Avner Reshef $^{1}{ }^{10}$ Donald Levy $^{2}$ Hilary Longhurst ${ }^{3}$ Marco Cicardi ${ }^{\dagger 4}$ Timothy Craig $^{5}$ Paul K. Keith ${ }^{6}$ Annette Feussner $^{7}$ Henrike Feuersenger ${ }^{7}$ Thomas Machnig $^{8} \quad$ Subhransu Prusty $^{7}$ Ingo Pragst ${ }^{7}$

Thromb Haemost 2021;121:690-693.

Replacement therapy with a plasma-derived subcutaneous C1-esterase inhibitor (pdC1-INH[SC]) has been approved for prophylaxis of hereditary angioedema (HAE) attacks., ${ }^{1,2}$ Since high levels of coagulation and fibrinolysis-related proteins have been previously reported in $\mathrm{HAE}^{3-6}$ we sought to evaluate the effects of the prophylactic treatment on measurable laboratory parameters. Twice weekly pdC1$\mathrm{INH}(\mathrm{SC}) 40$ or $60 \mathrm{IU} / \mathrm{kg}$ doses were administered in two clinical studies: phase 3 , randomized, double-blind, placebocontrolled, crossover trial (COMPACT) and Open-Label Extension (OLE) study. ${ }^{1,2}$ Continuous supplementation of pdC1-INH(SC) was found to be safe and effective in reducing attacks of HAE, while no related thromboembolic events were reported. Detailed design and results of both trials have been previously published. ${ }^{1,2}$ We report an analysis of plasma coagulation and fibrinolytic parameters recorded during prescheduled visits in both trials.

Analyses were done using standard laboratory methods performed by a central laboratory. Descriptive statistics were used (safety population was analyzed). No sample size

\footnotetext{
$\bar{\dagger}$ Co-author Marco Cicardi passed away on August 11, 2019 at the time of reviewing this communication.
}

Address for correspondence Avner Reshef, MD, Division of Allergy, Immunology, and Angioedema, Barzilai Medical Center, Ashkelon, Israel (e-mail: aresh@netvision.net.il).

calculation or statistical testing was performed for the coagulation endpoints.

We present here results for the Food and Drug Administration-approved pdC1-INH(SC) 60 IU/kg dose (HAEGARDA [United States and Canada] and Berinert SC [European Union and Australia]; CSL Behring) and placebo groups combined. Detailed demographics were previously published. ${ }^{1,2}$ Of the 90 patients in COMPACT, 60 (66.7\%) were females and the mean (standard deviation) age was 39.6 (14.9) years. Of the 126 patients in OLE, $76(60.3 \%)$ were females and the mean age was 40.5 (15.6) years. In both trials there were no notable differences in gender, race, and body mass index between pdC1-INH(SC) and placebo.

Although median values of all coagulation parameters remained within reference values at all time points assessed in both groups, median D-dimer (DD) and prothrombin fragments $1+2(\mathrm{PF} 1+2)$ in pdC1-INH(SC)-treated patients decreased from baseline throughout the study and up to week 14 during the COMPACT trial. Similar levels of DD and PF1 +2 were observed during the OLE trial, which remained stable (-Fig. 1). Such a trend was not observed in HAE patients receiving placebo. The median DD change (25th, 75th percentile) from baseline to week 14 was -120.0 $(-920.0,-10.0) \mathrm{ng} / \mathrm{mL}$ for pdC1-INH(SC) and $100.0(-20.0$, (c) 2020. Thieme. All rights reserved. Georg Thieme Verlag KG,

Rüdigerstraße 14,

70469 Stuttgart, Germany
DOI https://doi.org/ 10.1055/s-0040-1721147. ISSN 0340-6245. 

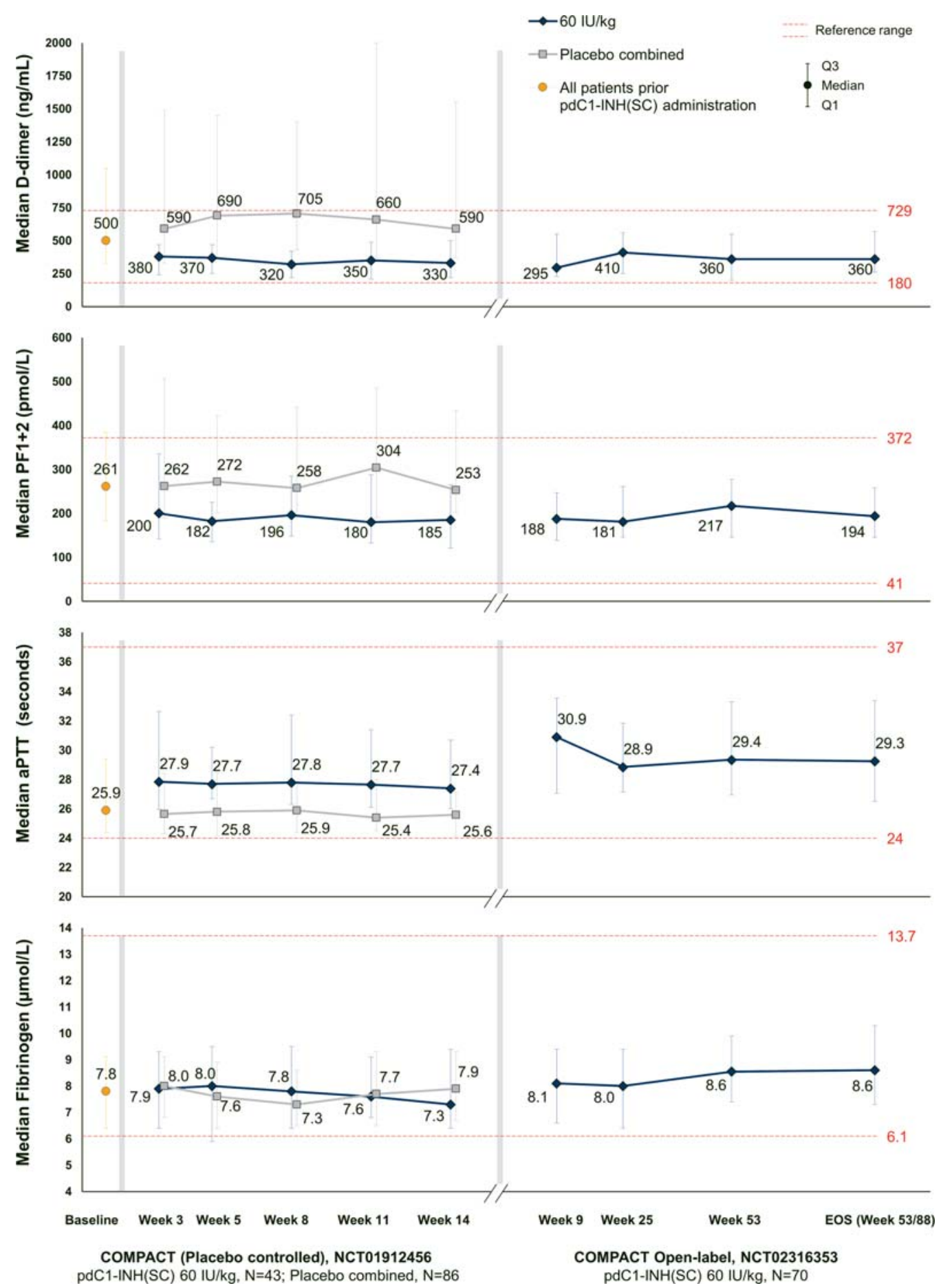

Fig. 1 Median D-dimer, PF1 + 2, aPTT, and fibrinogen levels (safety population). Notes: baseline values are for both COMPACT study and OLE (all patients prior $\mathrm{C} 1-\mathrm{INH}[\mathrm{SC}]$ administration). Red dotted lines represent normal laboratory range. EOS visit also includes values from the week 53 visit. aPTT, activated partial thromboplastin time; EOS, end of study; $\mathrm{N}$, number of patients; OLE, open-label extension; pdC1-INH(SC), plasmaderived subcutaneous C1-esterase inhibitor; PF1 + 2, prothrombin fragments $1+2$; Q1, first quartile (25th percentile); Q3, third quartile (75th percentile).

740.0) $\mathrm{ng} / \mathrm{mL}$ for placebo. The median PF1 +2 change from baseline to week 14 was $-52.5(-148.0,-19.0) \mathrm{pmol} / \mathrm{L}$ for pdC1-INH(SC) and $28.0(-30.0,192.0) \mathrm{pmol} / \mathrm{L}$ for placebo. Extreme DD and PF1 +2 levels were recorded during both trials. However, no consequential-related thrombotic or thromboembolic events were reported during the studies. Activated partial thromboplastin time levels were higher in treated patients but remained stable throughout both trials (-Fig. 1). No clinically relevant differences between pdC1$\mathrm{INH}(\mathrm{SC})$-treated and placebo-treated patients were observed over time for fibrinogen $(\boldsymbol{- F i g . 1} \mathbf{1}$ ), prothrombin international normalized ratio (data not shown), and plasmin- $\alpha 2$-antiplasmin complexes (data not shown), and these values were generally stable over time. 
C1-INH is a key inhibitor of the coagulation and fibrinolysis in HAE. ${ }^{6-8}$ In its absence both the contact system (CS) and the kallikrein-kinin system (KKS) are mobilized into overproduction of the vasoactive substance bradykinin-the main mediator of hyperpermeability and tissue edema (-Fig. 2). An increase in coagulation and fibrinolytic activity has been previously demonstrated in patients during attacks. ${ }^{3,9-17}$ Remarkably, there is no evidence of increased thrombotic events in HAE patients, neither during the activated state (attacks) nor in remission. ${ }^{11,12}$ One explanation is that generation of plasmin and mobilization of the fibrinolytic cascade may counterbalance clot formation. ${ }^{17}$ Additionally, it was shown that factor XII-dependent KKS may be activated without activating the factor XI-dependent intrinsic coagulation cascade, ${ }^{1-6,9-15}$ which may explain why the extent of thrombin generation during an attack is mild and self-limiting. ${ }^{9}$ Another explanation is that unlike the CS, which depends on factor XII autoactivation, the KKS may be activated in a factor XII-independent (contactless) manner on endothelial surfaces. ${ }^{14,15}$

We previously reported significantly elevated DD levels in HAE patients who experienced attacks with submucosal involvement. ${ }^{12}$ This may suggest that DD forming mechanisms may differ between tissues and could reflect differences in endothelial involvement. Since production of DD depends on broken-down insoluble cross-linked fibrin clots, it is still unclear where the high levels of fibrin seen during HAE attacks are originated.
We propose that in C1-INH-deficient patients the potential prothrombotic propensity is counterbalanced by increased fibrinolysis, manifested by high DD and PF1 + 2 plasma levels. Patients in COMPACT and OLE trials had frequent attacks and showed high DD and PF1 +2 at baseline and during placebo treatment. DD levels are used in clinical practice to categorize venous thromboembolism into highly or moderately sensitive diagnostic criteria. ${ }^{18}$ Very little is known about the significance of DD levels in chronic undulating diseases like HAE. Although still within the defined reference levels for thromboembolic risk, the study shows that these levels were attenuated during pdC1-INH(SC) continuous supplementation, which suggests that vascular homeostasis was restored.

Plasma coagulation and fibrinolysis differ in their plasma halflife (DD: 3-6 hours; PF1 + 2: 1.5 hours) ${ }^{19}$; therefore, their detection is dependent on the evaluation timing. This analysis carries this inherent limitation, since laboratory parameters were measured only during preset time points (patient visits), which does not allow comparing clinical attacks to remissions. However, the HAE patients included in both studies were considered highly active (having $\geq 4$ clinically significant attacks over a 2-month period before starting study treatment), ${ }^{1,2}$ which together with the proven effectiveness of the treatment underscores the relevance of the presented coagulation/fibrinolysis data.

Our results corroborate previous observations that C1INH-treated HAE patients show lower DD levels ${ }^{11,12}$ and that the transient elevation in coagulation parameters has not been associated with thromboembolic risk. Moreover, a

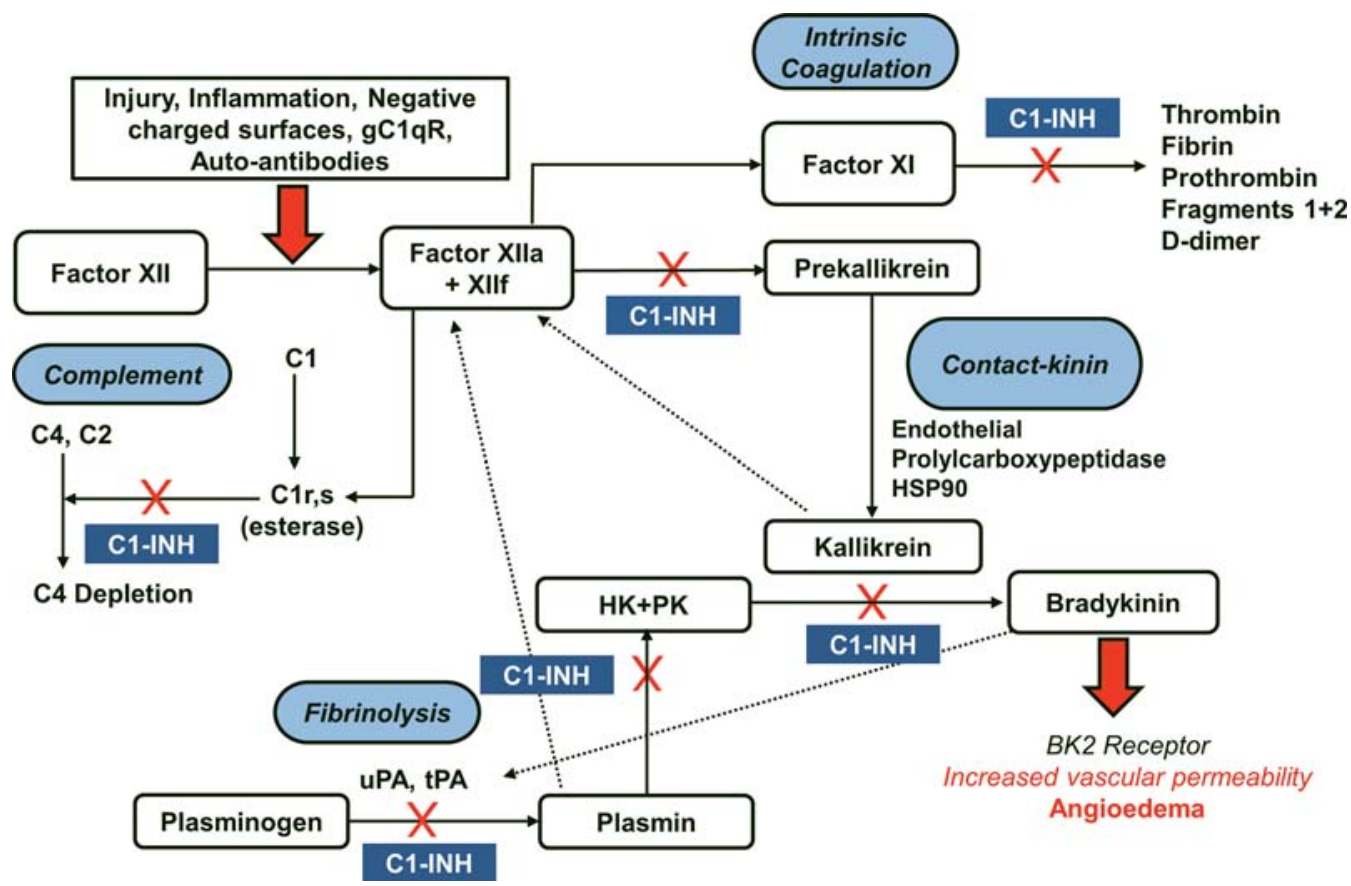

Fig. 2 Mechanism of action of C1-esterase inhibitor (C1-INH). In the absence/deficiency of the regulatory serine-protease inhibitor C1-INH, the contact activation system and the kallikrein-kinin system are activated by proteases associated with the vessel-wall endothelium. As a result, PK (complexed with HK) is converted to the proteolytic enzyme PKa, which cleaves HK and liberates the vasoactive nonapeptide BK. ${ }^{8}$ Factor XIla initiates the intrinsic cascade of coagulation, leading to fibrin formation. ${ }^{7}$ The role of the fibrinolytic system in hereditary angioedema was only recently highlighted. Evidently, both BK and PKa induce zymogenic plasminogen activators tPA and uPA, which trigger the fibrinolytic system, dissolve stabilized fibrin lattices, and release PF1 +2 and D-dimer into the circulation. ${ }^{6-8} \mathrm{BK}$, bradykinin; C1-INH, C1-esterase inhibitor; HK, highmolecular kininogen; HSP90, heat-shock protein 90; PF1 + 2, prothrombin fragments $1+2$; PK, plasma prekallikrein; PKa, plasma kallikrein; tPA, tissue-type plasminogen activator; uPA, urokinase-type plasminogen activator. 
comprehensive review confirmed pdC1-INH's safety regarding thromboembolic events. ${ }^{12}$ Thus, while C1-INH deficiency causes increased levels of coagulation factors, C1-INH prophylaxis may contribute to better system stability.

The clinical significance of these observations is yet unclear. Currently no laboratory marker has been accepted as a reliable predictor of HAE activity, ${ }^{16,20}$ although cleaved high-molecular kininogen has been proposed in some publications. ${ }^{20,21}$ Therefore, further research is warranted to explore the potential of DD and PF $1+2$ as HAE biomarkers. Our analysis supports DD as a surrogate marker for modification in disease activity achieved by prophylactic C1-INH supplementation.

\section{ClinicalTrials.gov Number \\ NCT01912456 (COMPACT) and NCT02316353 (OLE).}

\section{Authors' Contributions}

H.L., M.C., and T.C. were investigators in the COMPACT trial contributing to data generation. A.R., D.L., P.K.K., A.F., H.F., T.M., S.P., and I.P. reviewed and critically revised this communication. H.F. also provided statistical support. All authors approved the final version of this communication.

\section{Funding}

This work was funded by CSL Behring. Medical writing and editorial support were provided by Luis Araujo and Bhawna Basin of Trilogy Writing \& Consulting GmbH.

\section{Conflict of Interest}

A.R. has received research grant support to institution from CSL Behring, Shire HGT, Pharming, Stallergenes, BioCryst, and Teva. D.L. is a researcher, speaker, and consultant to CSL Behring, speaker to Takeda, and consultant to BioCryst. H.L. has received grant support, personal fees, and nonfinancial support from CSL Behring during the conduct of the trial, grant support from BioCryst and Takeda, personal fees from Adverum, BioCryst, Pharming, and Takeda, travel support from CSL Behring, and nonfinancial support from Pharming and Takeda. M.C. received grants from Shire and personal fees from Alnylam, BioCryst Pharmaceuticals, CSL Behring, Dyax, KalVista, Pharming Technologies, Shire, Sobi (Swedish Orphan Biovitrum), and ViroPharma. T.C. reports grant support from CSL Behring during the conduct of the trial. He is a speaker to CSL Behring, Takeda, and Grifols, a consultant to CSL Behring, Takeda, and BioCryst. He has received research support from CSL Behring, Takeda, and BioCryst. P.K.K. reports grant support from AstraZeneca, CSL Behring, Genentech, and Shire. All other authors declare no competing interests.

\section{References}

1 Longhurst H, Cicardi M, Craig T, et al; COMPACT Investigators. Prevention of hereditary angioedema attacks with a subcutaneous C1 inhibitor. N Engl J Med 2017;376(12):1131-1140

2 Craig T, Zuraw B, Longhurst $\mathrm{H}$, et al; COMPACT Investigators. Longterm outcomes with subcutaneous C1-inhibitor replacement therapy for prevention of hereditary angioedema attacks. J Allergy Clin Immunol Pract 2019;7(06):1793.e2-1802.e2

3 Cugno M, Zanichelli A, Bellatorre AG, Griffini S, Cicardi M. Plasma biomarkers of acute attacks in patients with angioedema due to C1-inhibitor deficiency. Allergy 2009;64(02):254-257

4 Deroux A, Dumestre-Perard C, Khalil-Mgharbel A, et al. BIOBRAD study: the search for biomarkers of bradykinin-mediated angiooedema attacks. Int Arch Allergy Immunol 2016;170(02): 108-114

5 Kaplan AP, Maas C. The search for biomarkers in hereditary angioedema. Front Med (Lausanne) 2017;4:206

6 Kaplan AP. Enzymatic pathways in the pathogenesis of hereditary angioedema: the role of $\mathrm{C} 1$ inhibitor therapy. J Allergy Clin Immunol 2010;126(05):918-925

7 Csuka D, Veszeli N, Imreh É, et al. Comprehensive study into the activation of the plasma enzyme systems during attacks of hereditary angioedema due to C1-inhibitor deficiency. Orphanet J Rare Dis 2015;10:132

8 Kaplan AP, Joseph K. Complement, kinins, and hereditary angioedema: mechanisms of plasma instability when $\mathrm{C} 1$ inhibitor is absent. Clin Rev Allergy Immunol 2016;51(02):207-215

9 van Geffen M, Cugno M, Lap P, Loof A, Cicardi M, van Heerde W. Alterations of coagulation and fibrinolysis in patients with angioedema due to C1-inhibitor deficiency. Clin Exp Immunol 2012;167(03):472-478

10 Gandhi PK, Gentry WM, Bottorff MB. Thrombotic events associated with $\mathrm{C} 1$ esterase inhibitor products in patients with hereditary angioedema: investigation from the United States Food and Drug Administration adverse event reporting system database. Pharmacotherapy 2012;32(10):902-909

11 Cugno M, Cicardi M, Bottasso B, et al. Activation of the coagulation cascade in C1-inhibitor deficiencies. Blood 1997;89(09): 3213-3218

12 Reshef A, Zanichelli A, Longhurst H, Relan A, Hack CE. Elevated D-dimers in attacks of hereditary angioedema are not associated with increased thrombotic risk. Allergy 2015;70(05): 506-513

13 Konings J, Hoving LR, Ariëns RS, et al. The role of activated coagulation factor XII in overall clot stability and fibrinolysis. Thromb Res 2015;136(02):474-480

14 Schmaier AH. The contact activation and kallikrein/kinin systems: pathophysiologic and physiologic activities. J Thromb Haemost 2016;14(01):28-39

15 De Maat S, Hofman ZLM, Maas C. Hereditary angioedema: the plasma contact system out of control. J Thromb Haemost 2018;16 (09):1674-1685

16 Defendi F, Charignon D, Ghannam A, et al; National Reference Centre for Angioedema CREAK. Enzymatic assays for the diagnosis of bradykinin-dependent angioedema. PLoS One 2013;8(08): e70140

17 Nielsen EW, Johansen HT, Høgåsen K, Wuillemin W, Hack CE, Mollnes TE. Activation of the complement, coagulation, fibrinolytic and kallikrein-kinin systems during attacks of hereditary angioedema. Scand J Immunol 1996;44(02):185-192

18 Kearon C. Diagnosis of suspected venous thromboembolism. Hematology (Am Soc Hematol Educ Program) 2016;2016(01): 397-403

19 Adcock DM, Bethel MA, Macy PA. Coagulation Handbook. Austin, TX: Esoterix Coagulation; 2006

20 Suffritti C, Zanichelli A, Maggioni L, Bonanni E, Cugno M, Cicardi M. High-molecular-weight kininogen cleavage correlates with disease states in the bradykinin-mediated angioedema due to hereditary C1-inhibitor deficiency. Clin Exp Allergy 2014;44(12): 1503-1514

21 Baroso R, Sellier P, Defendi F, et al. Kininogen cleavage assay: diagnostic assistance for kinin-mediated angioedema conditions. PLoS One 2016;11(09):e0163958 